\title{
Time-Like Baryon Transitions studies with HADES
}

B. Ramstein ${ }^{1, *}$ for the HADES Collaboration

J. Adamczewski-Musch ${ }^{4}$, O. Arnold ${ }^{10,9}$, B. Arnoldi-Meadows ${ }^{8}$, A. Belounnas ${ }^{1}$, A. Belyaev ${ }^{7}$, J. Biernat ${ }^{3}$, A. Blanco ${ }^{17}$, C. Blume ${ }^{8}$, M. Böhmer ${ }^{10}$, P. Bordalo ${ }^{17}$, L. Chlad ${ }^{18}$, P. Chudoba $^{18}$, I. Ciepał ${ }^{2}$, C. Deveaux ${ }^{11}$, D. Dittert ${ }^{5}$, J. Dreyer ${ }^{6}$, L. Fabbietti ${ }^{10,9}$, O. Fateev ${ }^{7}$, P. Fonte ${ }^{1, a}$, C. Franco ${ }^{17}$, J. Friese ${ }^{10}$, I. Fröhlich ${ }^{8}$, T. Galatyuk ${ }^{5,4}$, J. A. Garzón ${ }^{19}$, R. Gernhäuser ${ }^{10}$, A. Gillitzer ${ }^{12}$, M. Golubeva ${ }^{13}$, R. Greifenhagen ${ }^{6, c}$, F. Guber ${ }^{13}$, M. Gumberidze ${ }^{5, b}$, S. Harabasz ${ }^{5,3}$, T. Heinz ${ }^{4}$, T. Hennino ${ }^{1}$, M. Himmelreich ${ }^{8}$, C. Höhne ${ }^{11}$, R. Holzmann ${ }^{4}$, A. Ierusalimov ${ }^{7}$, V. Ivanov ${ }^{7}$, A. Ivashkin ${ }^{13}$, B. Kämpfer ${ }^{6, c}$, M. Kajetanowicz ${ }^{3}$, K-H. Kampert ${ }^{21}$, T. Karavicheva ${ }^{13}$, B. Kardan ${ }^{8}$, V. Khomyakov ${ }^{14}$, I. Koenig ${ }^{4}$, W. Koenig ${ }^{4}$, G. Korcyl ${ }^{3}$, G. Kornakov ${ }^{5}$, F. Kornas ${ }^{5}$, R. Kotte ${ }^{6}$, A. Kozela ${ }^{2}$, J. Kuboś ${ }^{2}$, A. Kugler ${ }^{18}$, T. Kunz ${ }^{10}$, A. Kurepin ${ }^{13}$, P. Kurilkin ${ }^{7}$, V Kushpil ${ }^{18}$, V. Ladygin ${ }^{7}$, R. Lalik ${ }^{10,9}$, A. Lebedev ${ }^{14}$, S. Linev ${ }^{4}$, M. Liu ${ }^{11}$, L. Lopes ${ }^{17}$, M. Lorenz ${ }^{8}$, G. Lykasov ${ }^{7}$, T. Mahmoud ${ }^{11}$, A. Malakhov ${ }^{7}$, J. Markert ${ }^{4}$, S. Maurus ${ }^{10}$, V. Metag ${ }^{11}$, J. Michel ${ }^{8}$, D.M. Mihaylov ${ }^{10,9}$, V. Mikhaylov ${ }^{18}$, S. Morozov ${ }^{13,15}$, C. Müntz ${ }^{8}$, L. Naumann ${ }^{6}$, K. Nowakowski ${ }^{3}$, Y. Parpottas ${ }^{16, d}$, V. Patel ${ }^{21}$, C. Pauly ${ }^{21}$, V. Pechenov ${ }^{4}$, O. Pechenova ${ }^{8}$, A. Pereira ${ }^{17}$, V. Petousis ${ }^{16}$, O. Petukhov ${ }^{13,15}$, D. Pfeifer ${ }^{21}$, J. Pietraszko ${ }^{4}$, W. Przygoda ${ }^{3}$, K. Pysz ${ }^{2}$, S. Ramos ${ }^{17}$, A. Reshetin ${ }^{13}$, L. Ritman ${ }^{12}$, P. Rodriguez-Ramos ${ }^{18}$, A. Rost ${ }^{5}$, A. Sadovsky ${ }^{13}$, P. Salabura ${ }^{3}$, T. Scheib ${ }^{8}$, H. Schuldes ${ }^{8}$, E. Schwab ${ }^{4}$, F. Scozzi ${ }^{5,1}$, F. Seck ${ }^{5}$, P. Sellheim ${ }^{8}$, I. Selyuzhenkov ${ }^{15,4}$, L. Silva ${ }^{17}$, J. Smyrski ${ }^{3}$, M. Sobiella ${ }^{6}$, S. Spataro ${ }^{20}$, S. Spies ${ }^{8}$, H. Ströbele ${ }^{8}$, J. Stroth ${ }^{8,4}$, P. Strzempek ${ }^{3}$, O. Svoboda ${ }^{18}$, M. Szala $^{8}$, A. Taranenko ${ }^{15}$, P. Tlusty ${ }^{18}$, M. Traxler ${ }^{4}$, A. Troyan ${ }^{7}$, H. Tsertos ${ }^{16}$, V. Wagner ${ }^{18}$, C. Wendisch ${ }^{4}$, M.G. Wiebusch ${ }^{8}$, P. Wintz ${ }^{12}$, J. Wirth ${ }^{10,9}$, B. Włoch ${ }^{2}$, A. Zhilin ${ }^{14}$, A. Zinchenko ${ }^{7}$, P. Zumbruch ${ }^{4}$, M. Zuschke ${ }^{8}$

\footnotetext{
${ }^{1}$ Institut de Physique Nucléaire, CNRS-IN2P3, Univ. Paris-Sud, Univ. Paris-Saclay, 91406 Orsay, France

${ }^{2}$ Institute of Nuclear Physics, Polish Academy of Sciences, 31342 Kraków, Poland

${ }^{3}$ Smoluchowski Institute of Physics, Jagiellonian University of Cracow, 30-059 Kraków, Poland

${ }^{4}$ GSI Helmholtzzentrum für Schwerionenforschung GmbH, 64291 Darmstadt, Germany

${ }^{5}$ Technische Universität Darmstadt, 64289 Darmstadt, Germany

${ }^{6}$ Institut für Strahlenphysik, Helmholtz-Zentrum Dresden-Rossendorf, 01314 Dresden, Germany

${ }^{7}$ Joint Institute of Nuclear Research, 141980 Dubna, Russia

${ }^{8}$ Institut für Kernphysik, Goethe-Universität, 60438 Frankfurt, Germany

${ }^{9}$ Excellence Cluster 'Origin and Structure of the Universe', 85748 Garching, Germany

${ }^{10}$ Physik Department E62, Technische Universität München, 85748 Garching, Germany

${ }^{11}$ II.Physikalisches Institut, Justus Liebig Universität Giessen, 35392 Giessen, Germany

${ }^{12}$ Forschungszentrum Juelich, 52428 Juelich, Germany

${ }^{13}$ Institute for Nuclear Research, Russian Academy of Science, 117312 Moscow, Russia

${ }^{14}$ Institute of Theoretical and Experimental Physics, 117218 Moscow, Russia

${ }^{15}$ National Research Nuclear University MEPhI (Moscow Engineering Physics Institute), 115409 Moscow, Russia

${ }^{16}$ Department of Physics, University of Cyprus, 1678 Nicosia, Cyprus

${ }^{17}$ LIP-Laboratório de Instrumentação e Física Experimental de Partículas, 3004-516 Coimbra,
}

*e-mail: ramstein@ipno.in2p3.fr 


\author{
Portugal \\ ${ }^{18}$ Nuclear Physics Institute, The Czech Academy of Sciences, 25068 Rez, Czech Republic \\ ${ }^{19}$ LabCAF. F. Física, Univ. de Santiago de Compostela, 15706 Santiago de Compostela, Spain \\ ${ }^{20}$ Dipartimento di Fisica and INFN, Università di Torino, 10125 Torino, Italy \\ ${ }^{21}$ Bergische Universität Wuppertal, 42119 Wuppertal, Germany \\ ${ }^{a}$ also at ISEC Coimbra, Coimbra, Portugal \\ ${ }^{b}$ also at ExtreMe Matter Institute EMMI, 64291 Darmstadt, Germany \\ ${ }^{c}$ also at Technische Universität Dresden, 01062 Dresden, Germany \\ ${ }^{d}$ also at Frederick University, 1036 Nicosia, Cyprus
}

\begin{abstract}
The HADES collaboration uses the $\mathrm{e}^{+} \mathrm{e}^{-}$production as a probe of the resonance matter produced in collisions at incident energies of $1-3.5 \mathrm{GeV} / \mathrm{nu}$ cleon at GSI. Elementary reactions provide useful references for these studies and give information on resonance Dalitz decays $\left(\mathrm{R} \rightarrow \mathrm{Ne}^{+} \mathrm{e}^{-}\right)$. Such processes are sensitive to the structure of time-like electromagnetic baryon transitions in a kinematic range where (off-shell) vector mesons play a crucial role. Results obtained in proton-proton reactions and in a commissioning pion-beam experiment are reported and prospects for future pion beam experiments and for first hyperon Dalitz decay measurements are described. The connection with the investigations of medium effects to be continued with HADES in the next years at SIS18 and SIS100 is also discussed.
\end{abstract}

\title{
1 Introduction
}

The main objective of experiments with the High Acceptance Di-Electron Spectrometer (HADES) $[1,2]$ at GSI is the study of hadronic matter in the low temperature $(\mathrm{T}<80 \mathrm{MeV})$ and large density (up to 3 times the normal nuclear matter density) region. The study of this part of the QCD phase diagram attracts an increasing interest, because of its similarity with the hadronic matter produced in the collision of dense stellar objects. In addition, exotic phenomena are predicted [3], which need to be checked experimentally. Dielectrons are used as one of the favored probes for hadronic matter studies, to investigate in particular in-medium vector meson spectral functions, which are expected to be modified, due to the coupling of $\rho$ mesons to resonance-hole states [4].

The HADES experimental program using elementary reactions (pp, "quasifree" np and more recently $\pi \mathrm{N}$ reactions) provide useful reference spectra for the interpretation of medium effects. In particular, the role of the $\rho$ mesons in the Dalitz decays of baryonic resonances $\left(\mathrm{R} \rightarrow \mathrm{Ne}^{+} \mathrm{e}^{-}\right)$is investigated. By studying these processes, insight can be gained into the electromagnetic structure of baryonic transitions in the time-like region at small four-momentum squared $M_{e e}^{2}=\mathrm{q}^{2} \in\left[4 m_{e}^{2},\left(M_{B}-M_{N}\right)^{2}\right]$, where $M_{e e}, m_{e}, M_{B}$ and $M_{N}$ are the electron, resonance and nucleon masses, respectively. The ultimate goal of studying the Dalitz decays is to extract $\mathrm{q}^{2}$ dependent electromagnetic form factors or equivalently helicity amplitudes [5] in the time-like region for individual baryon transitions, to get similar information as the one provided in the space-like region using electron scattering experiments [6]. This contribution is organized as follows. Some results from nucleon-nucleon and from pion-induced reactions are described respectively in Sec. 2 and Sec. 3. Prospects for future experiments, in the context of the FAIR Phase-0 program to study hyperon Dalitz decays are discussed in Sec. 4. Finally, Sec. 5 deals with the connection to the medium effects studies. 


\section{Selected results from pp reactions}
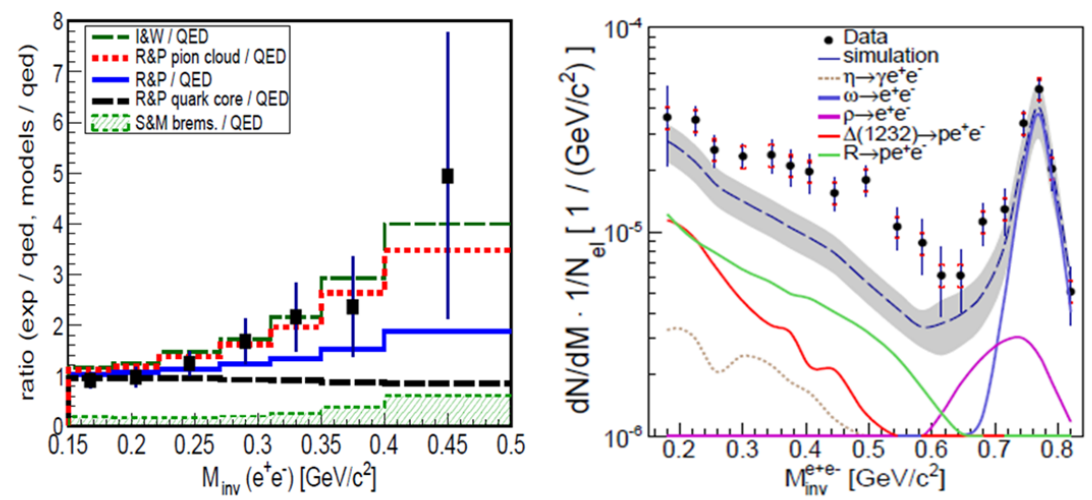

Figure 1. Left: Ratio of the $\mathrm{e}^{+} \mathrm{e}^{-}$invariant mass distribution in the $p p \rightarrow p p \mathrm{e}^{+} \mathrm{e}^{-}$reaction at $1.25 \mathrm{GeV}$ expected for a point-like ('QED') transition and the predictions with form factor models [7] (I\&W) (dashed dark green) and [8] (R\&P) (full blue: total, dashed red: meson cloud contribution, dashed black: quark core contribution) (see [9]). Right: Dielectron invariant mass distribution in the $p p \rightarrow p p \mathrm{e}^{+} \mathrm{e}^{-} \mathrm{X}$ reaction at an incident energy of $3.5 \mathrm{GeV}$ compared to simulation results. For the baryon Dalitz decay (green curve), point-like $B N \gamma^{*}$ couplings are used and the resonance production cross sections are fixed using pionic channels. For details, see [10].

Using pp reactions at an incident energy of $1.25 \mathrm{GeV}$, the branching ratio of the $\Delta(1232)$ Dalitz decay $\left(\Delta \rightarrow \mathrm{e}^{+} \mathrm{e}^{-}\right)$could be measured for the first time [9] and has been subsequently included in the review of the Particle Data Group [11]. The extracted value $B R=4.19 \pm 0.62$ (syst) \pm 0.34 (stat) $\times 10^{-5}$ is compatible with a calculation using constant form factors values fixed at $\mathrm{q}^{2}=0$. Nevertheless, the $\mathrm{e}^{+} \mathrm{e}^{-}$invariant mass spectrum shows some sensitivity to time-like $\mathrm{N}-\Delta(1232)$ transition form factors $[8,12]$ close to the kinematical limit $\left(q^{2}>0.1\right.$ $\mathrm{GeV}^{2}$ ) (fig. 1 Left). The best agreement is obtained in the model of [8], by considering only the meson cloud component which couples to the virtual photon either directly or via a virtual $\rho$ meson. The non-resonant pp bremsstrahlung contribution was estimated using OBE models $[13,14]$ and the $\Delta(1232)$ production amplitude was constrained by the Partial Wave Analysis (PWA) of the one-pion production channels [15].

A significant excess of the $\mathrm{e}^{+} \mathrm{e}^{-}$yield measured in the np reaction at the same energy with respect to the expected baryon contribution is observed $[16,17]$. It explains a long standing puzzle related to the large $\mathrm{e}^{+} \mathrm{e}^{-}$yield observed in light ion systems. While contributions from $n p \rightarrow d \mathrm{e}^{+} \mathrm{e}^{-}$process can contribute to the inclusive production $[18,19]$, the detailed analysis of the $\mathrm{pn} \rightarrow \mathrm{pne}^{+} \mathrm{e}^{-}$reaction shows results consistent with interpretation based on the effect of time-like pion electromagnetic form factor in charged currents, associated either to the non-resonant pn bremsstrahlung [18] or to the $\Delta \Delta$ interaction [20].

In the pp reactions at 2.2 and $3.5 \mathrm{GeV}$, the effect of time-like transition form factors of the Vector Dominance Type was clearly observed ([21] and references therein) as an excess of dielectron production for invariant masses below the vector meson pole mass with respect to calculations including constant (point-like) $\mathrm{RN} \gamma^{*}$ couplings. A detailed study of the baryonic resonance contribution was performed for pp collisions at $3.5 \mathrm{GeV}$ by combining the exclusive one pion production $\left(p p \rightarrow p p \pi^{0}\right.$ and $\left.p p \rightarrow p n \pi^{+}\right)$and dielectron $\left(p p \rightarrow p p \mathrm{e}^{+} \mathrm{e}^{-}\right)$ channels [10]. An analysis of the $\mathrm{pp} \rightarrow \mathrm{pp} \pi^{+} \pi^{-}$reaction at $3.5 \mathrm{GeV}$ is on-going, as described 
in more details in a contribution at this conference [22], which, in addition to single-baryon excitation, provides interesting information on double resonance excitation, off-shell and onshell $\rho$ production.

\section{Pion beam experiments}

One important advantage of studying baryon Dalitz decays with pion-nucleon interactions with respect to proton-proton collisions is that, in the dominant s-channel process, resonances are produced at a fixed mass, hence reducing the overlap between resonances of different masses. In addition, since the $\pi N \rightarrow N \gamma^{*}$ reaction and the $\gamma^{*} N \rightarrow N \pi$ reactions are related by crossing symmetry, inputs from known pion photo- or electroproduction amplitudes can be directly used to fix $\mathrm{e}^{+} \mathrm{e}^{-}$production amplitudes. Besides, the simultaneous measurement of hadronic channels can complement the very poor existing data base for pion induced reactions. The combination of the GSI secondary pion beam covering momentum range between 0.6 and $1.7 \mathrm{GeV} / \mathrm{c}$, corresponding to baryon masses of 1.43 to $2 \mathrm{GeV} / \mathrm{c}^{2}$ with the multipurpose HADES detector represents a unique facility in the world and the new HADES program with the pion beam is of special interest in view of existing plans for meson beam facilities for baryon spectroscopy at J-PARC [23] or EIC [24].

Recently, the GSI pion beam line, including beam tracking detectors [25] was used by the HADES collaboration in a commissioning experiment, exploring two different physics topics: the study of strangeness production and absorption in cold matter in $\pi+\mathrm{C}$ and $\pi+\mathrm{W}$ reactions at an incident momentum of $1.7 \mathrm{GeV} / \mathrm{c}$, as presented in this conference [26] and the measurement of hadronic and electromagnetic baryon couplings in the region of the $\mathrm{N}(1520)$ resonance, using a pion beam with momentum around $0.7 \mathrm{GeV} / \mathrm{c}$. For the latter study, a long data taking period was devoted to $\mathrm{e}^{+} \mathrm{e}^{-}$production measurement, using a polyethylene target $\left(\mathrm{C}_{2} \mathrm{H}_{4}\right)$ and a pion beam with a momentum of $0.69 \mathrm{GeV} / \mathrm{c}$. Three other measurements with smaller statistics were also performed at pion beam momenta of $0.656,0.748$ and 0.8 $\mathrm{GeV} / \mathrm{c}$ in order to provide new data on double pion production for Partial Wave Analysis. Measurements with a carbon target were also realized simultaneously, which allowed for a precise subtraction of interactions with carbon nuclei in the polyethylene target in the case of pion production channels, but not for electromagnetic channels, due to the limited statistics of the carbon target measurement. Differential distributions with an unprecedented precision were provided in the $\pi^{+} \pi^{-} n$ and $\pi^{0} \pi^{-} p$ channels and were included in a recent PWA in order to extract branching ratios of $\mathrm{N}(1440), \mathrm{N}(1520), \mathrm{N}(1535), \ldots$ in the different $2 \pi$ channels $(\rho \mathrm{N}$, $\sigma N, \Delta \pi)$ (see [27] and [28] at this conference).

The $\mathrm{e}^{+} \mathrm{e}^{-}$invariant mass distribution measured in the inclusive $\mathrm{e}^{+} \mathrm{e}^{-}$production in the $\pi+\mathrm{C}_{2} H_{4}$ target is presented in the left part of Fig. 2. The prominent peak at low $\mathrm{e}^{+} \mathrm{e}^{-}$invariant masses is due to the $\pi^{0}$ Dalitz decay $\left(\pi^{0} \rightarrow \gamma \mathrm{e}^{+} \mathrm{e}^{-}\right)$. In addition, the $\eta$ Dalitz decay has a significant contribution, arising from interactions on carbon nuclei due to the Fermi momentum. These mesonic contributions can be suppressed by applying $\mathrm{e}^{+} \mathrm{e}^{-}$invariant mass $\left(\mathrm{M}_{e e}>0.140 \mathrm{GeV} / \mathrm{c}^{2}\right)$ and missing mass $\left(0.9<M M<1.04 \mathrm{GeV} / \mathrm{c}^{2}\right)$ cuts which select events

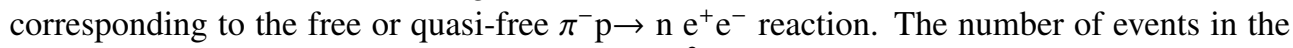
missing mass range between 0.9 and $1.04 \mathrm{GeV} / \mathrm{c}^{2}$ amounts to about 1500 , with an estimated contribution of about $66 \%$ of quasi-free $\pi^{-} \mathrm{p}$ interactions in the carbon nucleus. The $\mathrm{e}^{+} \mathrm{e}^{-}$ invariant mass spectrum presents a significant excess above the contribution of baryon Dalitz decays calculated for point-like objects, which signals electromagnetic form factors of the Vector Dominance Model type. Interestingly, this excess is consistent with the electromagnetic decay of off-shell $\rho \mathrm{s}$, as deduced from the PWA of the $2 \pi$ channels.

Further information can be deduced from the analysis of the angular distributions (right part of Fig. 2). The density matrix formalism [29] provides a convenient parametrization of 

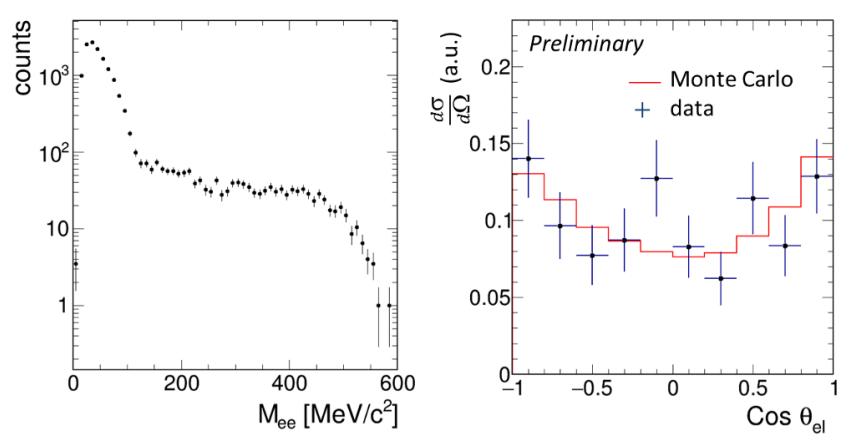

Figure 2. Dilepton production in the $\pi^{-}+\mathrm{CH}_{2}$ reaction at $0.69 \mathrm{GeV} / \mathrm{c}$. Left: inclusive $\mathrm{e}^{+} \mathrm{e}^{-}$invariant mass distribution. Right: The exclusive $\pi^{-} \mathrm{p} \rightarrow \mathrm{ne}^{+} \mathrm{e}^{-}$reaction is selected using missing mass. The distribution of the lepton angle $\theta_{e l}$ in the $\gamma^{*}$ reference frame is shown for $\gamma^{*}$ angles in the center-of-mass in the bin $0<\cos \left(\theta_{\gamma^{*}}^{C M}\right)<0.5$. The red curve represents the result of the fit weighted by acceptance and efficiency.

the amplitudes $|A|$ at a given value of $\mathrm{M}_{e e}$ and emission angle of the virtual photon, with three independent coefficients, that can be extracted from a fit. The results could be compared to predictions of models, like for example the recent Lagrangian model of [29]. While the electromagnetic form factors for individual transitions can not be extracted yet from this analysis, these results constitute the very first information about the baryon electromagnetic transitions in the time-like region and can be compared to model predictions, and in particular, check the validity of the VDM approach in the electromagnetic channels.

The HADES collaboration plans to use the same procedure in future experiments in the $\sqrt{s}=1.7 \mathrm{GeV} / \mathrm{c}^{2}$ region, where the emission of $\mathrm{e}^{+} \mathrm{e}^{-}$pairs, of real photons, thanks to a new Electromagnetic Calorimeter based on lead crystals that has just been installed, and various mesons $\left(\pi^{0}, \omega, \eta\right.$, kaons, ...) will be measured. These experiments will also benefit from the enhanced RICH detector efficiency provided by the new CSI photon detector. The aim is to measure about $10^{4} \mathrm{e}^{+} \mathrm{e}^{-}$pairs with mass larger than the $\pi^{0}$ mass to have enough statistics for a accurate analysis of invariant mass and angular distributions. In further future, the region of $\sqrt{s}=2 \mathrm{GeV} / \mathrm{c}^{2}$ can also be investigated to focus on $\Lambda(1405)$.

\section{Future hyperon studies}

Only scarce information exist on the electromagnetic structure of hyperons. Elastic electromagnetic factor factors have been measured in $\mathrm{e}^{+} \mathrm{e}^{-} \rightarrow Y \bar{Y}$ reactions by BABAR for $\mathrm{Y}=\Lambda^{0}$ and $\Sigma^{0}$ for $\mathrm{q}^{2}$ between the threshold and $9 \mathrm{GeV}^{2}$ and by CLEO for $\mathrm{Y}=\Lambda^{0}, \Sigma^{0,+}, \Xi^{0,+}, \Omega^{-}$at $\mathrm{q}^{2}=14$ and $17.4 \mathrm{GeV}^{2}$, showing a sensitivity to quark correlations and new measurements from BES III are being analyzed ([30] and references therein). After 2020, the HADES collaboration plans to start a program to measure hyperon radiative and Dalitz decays ( $\mathrm{Y}^{\prime} \rightarrow \mathrm{Y} \gamma$, $\mathrm{Y}^{\prime} \rightarrow \mathrm{Ye}^{+} \mathrm{e}^{-}$) which will provide complementary information for low $\mathrm{q}^{2}$ in the time-like region. One open question is the relevance of VDM in the hyperon sector [31], which plays an important role in the non-strange baryon sector, as pointed out above. Since most of the hyperons are narrow, they can be identified by the invariant mass of their decay products and their abundant production in proton-proton reactions (about $130 \mu \mathrm{b}$ for $\Lambda(1520)$ at $4.5 \mathrm{GeV}$ ) or proton-nucleus reactions can be used. HADES can study their weak decays, as well as radiative and Dalitz decays of hyperons. These measurements will benefit from the on-going 
upgrade of the HADES set-up. In particular, the possibility to detect forward emitted protons with the new Straw Tube detector will be exploited and the Electromagnetic Calorimeter will be essential for the measurement of radiative decays. The increased acquisition rate (up to $200 \mathrm{kHz}$ ) is also needed to record enough statistics for the rare Dalitz decays. As described in detail in a poster at this conference [32], dedicated simulations have been performed for the $\Lambda(1520) / \Lambda(1405) / \Sigma(1385) \rightarrow \Lambda \mathrm{e}^{+} \mathrm{e}^{-}$Dalitz decay. In four weeks, $4300 \Lambda \mathrm{e}^{+} \mathrm{e}^{-}$events with $\mathrm{e}^{+} \mathrm{e}^{-}$invariant mass above the $\pi^{0}$ mass can be selected after all analysis cuts. HADES studies will allow in particular to compare the results for SU(3) partners like $\mathrm{N}(1520)$ and $\Lambda(1520)$ or $\Sigma(1385)$ and $\Delta(1232)$ to get information about the sensitivity of baryon electromagnetic structure to the strange quark content.

Proton-proton reactions at $4.5 \mathrm{GeV}$ can also be used to elucidate the $\Xi^{-}(1321)$ production mechanism. An unexpectedly large sub-threshold production of the $\Xi^{-}$resonance has indeed been measured in $\mathrm{ArKCl}$ and $\mathrm{pNb}$ reactions ([2] and references therein) and reference measurements in the nucleon-nucleon system are needed to test possible explanations related to the contribution of high mass resonances [33]. The $\Xi^{-}$is identified via its decay into $\Lambda$ and $\pi^{-}$pair, with the subsequent decay of the $\Lambda$ into a p $\pi^{-}$pair. Assuming a cross section of 4.8 $\mu \mathrm{b}, 2.810^{6} \Xi^{-}$are expected in four weeks of beam time, with a S/B close to 1 , which will allow for an accurate measurement of differential distributions.

\section{Connection with the HADES hadronic matter program}

Experiments with elementary reactions are essential for the interpretation of medium effects which constitute the main goal of the HADES experiments [2]. The coupling of baryonic resonances to the $\rho$ meson is indeed an important ingredient of calculations of the emissivity of hadron resonance matter. The intrinsic structure of baryons in terms of meson cloud and quark core is also essential to understand how new states of matter can develop in high density conditions. Many other observables (strangeness, flow, particle correlations, fluctuations, $\cdots$ ) are also exploited to characterize the dense matter and are compared to results from experiments at higher energies at SPS, RHIC or LHC. After successful investigations of the $\mathrm{C}+\mathrm{C}$, $\mathrm{Ar}+\mathrm{KCl}, \mathrm{p}+\mathrm{Nb}$ reactions and more recently of the $\mathrm{Au}+\mathrm{Au}, \pi^{-}+\mathrm{W}$ and $\pi^{-}+\mathrm{C}$ systems [2], an experiment aiming at measuring the $\mathrm{Ag}+\mathrm{Ag}$ system at $1.65 \mathrm{GeV} /$ nucleon will be performed in the near future. Cold matter studies are also foreseen in pion and proton induced reactions in the next years. The possibility of HADES to measure low-momentum pairs is a great advantage to study medium effects, such as modifications of the $\rho$ meson spectral function and $\omega$ and $\phi$ meson absorption [2]. Thanks to the high performances and the versatility of the HADES set-up, other measurements like the dark photon search [34] have also been successfully addressed. With the adjunction of neutron detectors at backward angles, HADES will also be well suited to investigate nucleon configurations at high relative momentum related to Short Range Correlations in proton-nucleus reactions, a measurement which can be performed after 2020 simultaneously to cold nuclear matter studies. The HADES collaboration will then continue the investigations of the hadronic matter using the SIS100 beams up to 4-6 $\mathrm{GeV} /$ nucleon, in complement to the CBM set-up, which is optimized for higher energies.

The collaboration gratefully acknowledges the support by SIP JUC Cracow (Poland), 2017/26/M/ST2/00600; TU Darmstadt (Germany), VH-NG-823; GU Frankfurt (Germany), BMBF:05P15RFFCA, HIC for FAIR, ExtreMe Matter Institute EMMI; TU München, Garching (Germany), MLL München, DFG EClust 153, BmBF 05P15WOFCA; JLU Giessen (Germany), BMBF:05P12RGGHM; NRNU MEPhI Moscow Excellence project 02.a03.21.0005, MSERF 3.3380.2017/4.; 6 IPN, IN2P3/CNRS (France); NPI CAS Rez (Czech Republic), MSMT OP VVV CZ.02.1.01/0.0/0.0/16 013/0001677, MSMT LM2015049. 


\section{References}

[1] G. Agakichiev et al. (HADES collaboration), Eur. Phys. J. A 41, 243 (2009)

[2] M. Lorenz et al. (HADES collaboration), PoS CPOD2017, 016 (2018)

[3] A. Andronic et al., Nucl. Phys. A 837, 65 (2010)

[4] R. Rapp, J. Wambach, Eur. Phys. J. A 6, 415 (1999)

[5] M.I. Krivoruchenko, A. Faessler, Phys. Rev. D 65, 017502 (2002)

[6] I. Aznauryan et al., Int. J. Mod. Phys. E 22, 1330015 (2013)

[7] I. Fröhlich et al., PoS ACAT2007, 076 (2007)

[8] G. Ramalho et al., Phys. Rev. D 93, 033004 (2016)

[9] J. Adamczewski-Musch et al. (HADES), Phys. Rev. C 95, 065205 (2017)

[10] G.. Agakishiev et al. (HADES collaboration), Eur. Phys. J. A 50, 82 (2014)

[11] M. Tanabashi et al. (Particle Data Group), Phys. Rev. D 98, 030001 (2018)

[12] Q. Wan, F. Iachello, Int. J. Mod. Phys. A 20, 1846 (2005)

[13] R. Shyam, U. Mosel, Phys. Rev. C 82, 062201 (2010)

[14] L.P. Kaptari, B. Kämpfer, Nucl. Phys. A 764, 338 (2006)

[15] G. Agakishiev et al. (HADES Collaboration), Eur. Phys. J. A 51, 137 (2015)

[16] G. Agakishiev et al. (HADES Collaboration), Phys. Lett. B 690, 118 (2010)

[17] J. Adamczewski-Musch et al. (HADES Collaboration), Eur. Phys. J. A 53 (2017) 149

[18] B. Martemyanov, M. Krivoruchenko, A. Faessler, Phys. Rev. C 84, 047601 (2011)

[19] H. Kuc, Ph.D. thesis, Paris-Sud university, France (2014)

[20] M. Bashkanov, H. Clement, Eur. Phys. J. A 50, 107 (2014)

[21] G. Agakishiev et al. (HADES collaboration), Eur. Phys. J. A 50, 82 (2014)

[22] A. Belounnas et al. (HADES collaboration), this conference

[23] H. Sako et al., PoS INPC2016, 266 (2017)

[24] W.J. Briscoe et al., Eur. Phys. J. A 51, 129 (2015)

[25] J. Adamczewski-Musch et al. (HADES collaboration), Eur. Phys. J. A 53, 188 (2017)

[26] J. Wirth et al. (HADES collaboration), this conference

[27] I. Ciepal et al. (HADES collaboration), this conference

[28] V.V. Sarantsev, this conference

[29] E. Speranza, M. Zetenyi, B. Friman, Phys. Lett. B 764, 282 (2017)

[30] K. Schönning, C. Li, EPJ Web Conf. 137, 12002 (2017)

[31] C. Granados, S. Leupold, E. Perotti, Eur. Phys. J. A 53, 117 (2017)

[32] K. Nowakowski et al. (HADES collaboration), this conference

[33] M. Zétényi and G. Wolf, Phys. Lett. B 785 (2018) 226

[34] G. Agakishiev et al. (HADES collaboration), Phys. Lett. B 731, 265 (2014) 